\title{
Laser Ablation Electrospray Ionization Hydrogen/Deuterium Exchange Ambient Mass Spectrometry Imaging
}

\author{
Fred A. M. G. van Geenen, ${ }^{\dagger},{ }^{\dagger}$ Frank W. Claassen, $^{\dagger}$ Maurice C. R. Franssen, ${ }^{\dagger}{ }^{\circledR}$ Han Zuilhof, ${ }^{\dagger, \|_{\odot}}$
} and Michel W. F. Nielen $*,, \perp_{\odot}$

\author{
${ }^{\dagger}$ Laboratory of Organic Chemistry, Wageningen University, Stippeneng 4, 6708 WE Wageningen, The Netherlands \\ \$TI-COAST, Science Park 904, 1098 XH Amsterdam, The Netherlands \\ ${ }^{\S}$ Institute for Molecules and Materials, FELIX Laboratory, Radboud University, Toernooiveld 7c, 6525 ED Nijmegen, The \\ Netherlands \\ "School of Pharmaceutical Sciences and Technology, Tianjin University, 92 Weijin Road, Tianjin 300072, P.R. China \\ ${ }^{\perp}$ Wageningen Food Safety Research (WFSR), Wageningen University \& Research, P.O. Box 230, 6700 AE Wageningen, The \\ Netherlands
}

\section{Supporting Information}

ABSTRACT: Identification and confirmation of known as well as unknown (bio)chemical entities in ambient mass spectrometry (MS) and MS imaging (MSI) mostly involve accurate mass determination, often in combination with MS/ MS or $\mathrm{MS}^{\mathrm{n}}$ work flows. To further improve structural assignment, additional molecular information is required. Here we present an ambient hydrogen/deuterium exchange (HDX) laser ablation electrospray ionization (LAESI) MS method in which, apart from the accurate mass and MS/MS data, the number of exchangeable protons in (un)known molecules is obtained. While eventually presenting ambient HDX-LAESI-MSI, samples were not preincubated with deuterated solvents, but instead HDX occurred following fusion of ablated sample material with microdroplets generated by ESI of deuterated solvents. Therefore, the degree of HDX was first studied following ablation of nondeuterated sample solutions of melamine and monosaccharides. From these experiments, it was concluded that the set-up used could provide meaningful HDX data in support of molecular structure elucidation by significantly reducing the number of structure options from a measured elemental composition. This reduction was demonstrated with an unknown accurate $m / z$ value obtained in the analysis of an orange slice, reducing the possible number of molecular structures having the same elemental composition by $87 \%$ due to the number of $\mathrm{H} / \mathrm{D}$ exchanges observed. Next, deuterated and nondeuterated MS/MS experiments showed the number of exchangeable protons in the substructures from deuterated neutral losses in the product ion spectra, confirming the compound to be arginine. Finally, the potential of ambient HDX-LAESI-MSI was demonstrated by the imaging of (secondary) plant metabolites in a Phalaenopsis petal.

KEYWORDS: laser ablation electrospray ionization, hydrogen deuterium exchange, ambient mass spectrometry, structure elucidation, mass spectrometry imaging

\section{INTRODUCTION}

Mass spectrometry (MS) studies commonly aim to find the identity and/or quantity of molecules in a sample. In mass spectrometry imaging (MSI) the localization of molecules is studied, mainly in plant or animal tissue such as lipid profiles and the accumulations of drugs and their metabolites. ${ }^{1-4}$ Tissue samples often require laborious pretreatment steps and vacuum conditions in order to be compatible with matrixassisted laser desorption ionization (MALDI) MSI analysis. ${ }^{5}$ However, sample pretreatment can largely affect the outcome of MSI due to analyte losses and/or delocalization of molecules. ${ }^{6,7}$ Additionally, samples can be disrupted or damaged by vacuum conditions. ${ }^{8,9}$ In contrast, ambient MS allows the acquisition of mass spectra from samples in their native environment, without any sample pretreatment. ${ }^{10-13}$

Structural identification in ambient MS(I) is largely dependent on accurate mass measurements, often combined with MS/MS or $\mathrm{MS}^{\mathrm{n}}$ approaches. ${ }^{14}$ The accurate mass is acquired with high-resolution instruments, such as time-offlight and Orbitrap MS, and provides the elemental composition of (bio)molecules. ${ }^{15}$ This elemental composition usually still yields numerous candidates of chemical substances

Received: June 13, 2019

Accepted: November 20, 2019

Published: January 8, 2020 
in database searches. ${ }^{16} \mathrm{MS} / \mathrm{MS}$ approaches can then further elucidate the (bio)molecular structure, but with hundreds of molecular structure options this requires the availability of numerous standard substances and large spectral libraries thereof. $^{17}$ Alternatively, orthogonal methods like ion mobility (IMS) or online reactions can be used during ambient MS(I) data acquisition to obtain additional information for final structure elucidation, but these are not or only partly useful for elucidation of unknown entities and are typically applied for improved selectivity and signal-to-noise ratio in targeted analysis. ${ }^{18-21}$ The development of methods to obtain additional molecular structural information is thus of utmost importance for reducing the number of structure options and thereby increasing the certainty in, as well as the speed of, identification in ambient MS(I).

A well-known approach that may assist in the identification of unknown entities is hydrogen/deuterium exchange (HDX) MS. $^{22}$ HDX-MS is a method to obtain the number of exchangeable hydrogens in a molecule, present in, e.g., $-\mathrm{NH}$ and $-\mathrm{OH}$ moieties. The $\mathrm{m} / \mathrm{z}$ value of any (un)known molecule or fragment thereof will increase accordingly with each included deuteron, providing valuable information on the number of exchangeable protons. This information can then be used to reduce the number of structure options for identical elemental compositions, ${ }^{23}$ limiting the need for hundreds of MS/MS experiments on numerous standards for comparison. Common HDX procedures rely on prolonged exchange reactions by dissolving the samples or standards in deuterated solvents, ${ }^{24}$ the addition of a deuterated gas to trapped gasphase ions for exchange reactions inside the vacuum of the mass spectrometer ${ }^{25}$ or, classically, using a deuterated reaction gas in chemical ionization (CI). ${ }^{26}$ However, gas-phase HDX of trapped ions is hard to combine with the wide $m / z$ range of non-targeted MSI common in, e.g., spatially resolved metabolomics studies. CI, or desorption atmospheric pressure chemical ionization (DAPCI), is only compatible with volatiles and very low molecular weight molecules, limiting its use for surface and tissue analysis. ${ }^{27}$ Dissolving/diluting tissue samples with deuterated solvents causes delocalization of analytes and hampers spatial resolution in surface analysis. Hence, a combination of HDX with ambient MSI would be a valuable addition for structure identification in surface analysis, including tissue. Apart from an initial DART set-up for ambient gas-phase HDX, ambient MS has not been explored for HDX yet. ${ }^{28}$

Laser ablation electrospray ionization (LAESI) is an ambient MS ionization technique that can also be used for imaging. ${ }^{29}$ LAESI-MS applicability was demonstrated in the analysis of tissue, $^{30-34}$ food contaminants, ${ }^{35}$ synthetic materials, ${ }^{36}$ and single cells. ${ }^{37-39}$ Furthermore, LAESI-MS was proven viable for performing and/or monitoring online time-resolved reactions. $^{20,40,41}$ In LAESI-MS analysis, ablated sample material is continuously extracted by charged microdroplets that are produced by an orthogonally placed electrospray emitter, prior to MS analysis. In an HDX-LAESI-MS approach the ESI solvent can easily be replaced with a deuterated one, resulting in a simple ambient MS HDX method that would enable imaging without the need to expose the sample surface to deuterated solvents and with a higher spatial resolution than in DART. As shown in previous ESI studies, the problem of deuterium-hydrogen back-exchange would be minimal as a result of the continuous ESI spray directed towards the MS inlet. $^{42-44}$ Obviously, various deuterium donors might be exploited, although in the present work $\mathrm{D}_{2} \mathrm{O}$ was mostly used. HDX reactions of exchangeable hydrogens are expected to be rapid due to the liquid microdroplet environment and to be characterized by up to complete conversion for at least small molecules. $^{45}$

In this research, we report a novel ambient MS approach, viz. HDX-LAESI, for (bio)molecular structure elucidation and confirmation. First, the degree of HDX conversion was studied for nondeuterated standard solutions of small molecules and critically compared with literature. Next, the applicability of HDX-LAESI-IMS-MS in (bio)molecular structure identification was shown for biomolecules like arginine and oligosaccharides, detected directly from an orange slice. Arginine was investigated in full detail by HDX-LAESI-MS/ MS experiments. The deuterated neutral losses provided additional evidence of molecular (sub)structures. Finally, the feasibility and added-value of ambient HDX-LAESI-MSI in spatially resolved metabolomics was demonstrated by the imaging of arginine in an orange slice and secondary plant metabolites within and outside the purple pigment regions of a Phalaenopsis petal.

\section{EXPERIMENTAL SECTION}

Materials. Ultrapure water $\left(\mathrm{H}_{2} \mathrm{O}\right) 18.2 \mathrm{M} \Omega \times \mathrm{cm}^{-1}$ at 25 ${ }^{\circ} \mathrm{C}$ was freshly produced with a Millipore (Molsheim, France) Integral 3 system. Deuterium oxide $\left(\mathrm{D}_{2} \mathrm{O}\right) 99.9 \%$ atom $\mathrm{D}$, methanol- $d_{4}$ (MeOD) $99.8 \%+$ atom $\mathrm{D}$, melamine, and Larginine were purchased from Sigma-Aldrich (Zwijndrecht, The Netherlands). A new ampule of $\mathrm{D}_{2} \mathrm{O}$ was used for each analysis. An orange was obtained from a local supermarket. An orchid (Phalaenopsis) was purchased from a local plant store.

Ambient HDX-LAESI-MS. A Protea Biosciences (Morgantown, WV) LAESI DP-1000 system was coupled to a Waters (Manchester, U.K.) Synapt G2-S traveling wave ion mobility (TWIM) time of flight mass spectrometer (TOFMS) and used for all analyses. Masslynx v4.1 SCN 883 (Waters) was used to control the experimental settings of the Synapt G2-S, which was operated in positive ion TOFMS resolution mode (mass resolution approximately 18000 FWHM, mass accuracy 5 $\mathrm{ppm}$ ) with a scan time of $1 \mathrm{~s}$, source temperature $150{ }^{\circ} \mathrm{C}$, sample cone $40 \mathrm{~V}$, and source offset $80 \mathrm{~V}$. In the case of oligosaccharide analysis, TWIM was applied using the following experimental conditions: the IMS wave velocity was set at $1200 \mathrm{~m} / \mathrm{s}$ and wave height was $40 \mathrm{~V}$. Driftscope v2.7 (Waters) was used to individually select drift time regions corresponding to oligosaccharides (Figure S1). Selected drift time data-containing all $\mathrm{m} / \mathrm{z}$ information residing within the selection-were exported back into Masslynx and backgroundsubtracted. The LAESI DP-1000 system was equipped with a $2940 \mathrm{~nm}$ mid-IR laser and controlled by LAESI desktop software v.2.0.1.3 (Protea Biosciences). Sample solutions were put in a 96-well plate. At each well 35 laser pulses were applied at a repetition rate of $5 \mathrm{~Hz}$ to produce a plume of ablated sample material, which was continuously extracted with charged microdroplets generated from an orthogonally placed electrospray emitter prior to MS analysis. The electrospray solvent was either $\mathrm{H}_{2} \mathrm{O}$ or $\mathrm{D}_{2} \mathrm{O}$ at a flow rate of $3 \mu \mathrm{L} / \mathrm{min}$. Electrospray voltage was set at $\sim 4 \mathrm{kV}$ in order to have a stable signal. Background-subtracted mass spectra were generated using the "combine spectrum" function in Masslynx: 5 scans were combined and 20 scans of electrospray background were subtracted. 

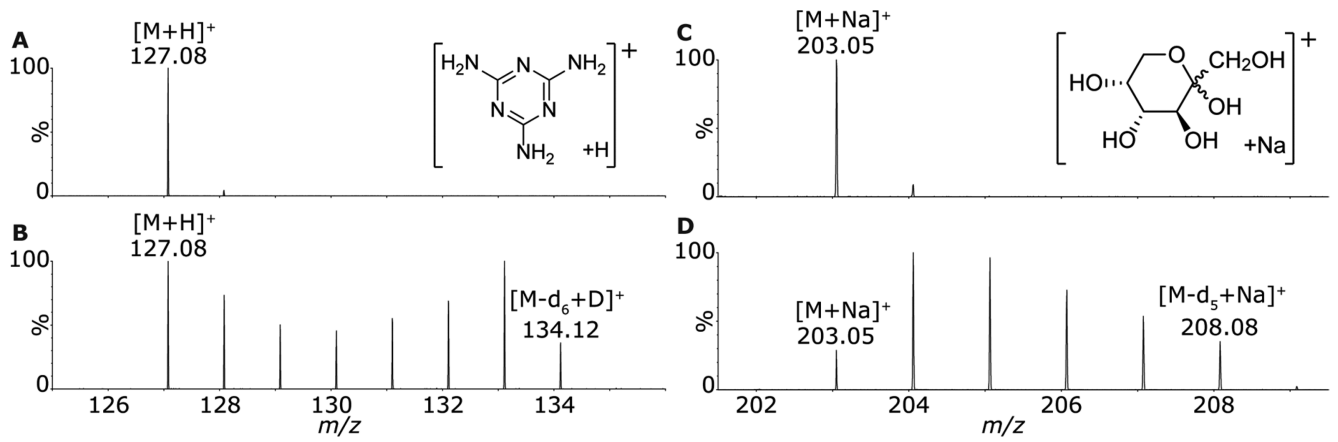

Figure 1. LAESI-MS and HDX-LAESI-MS of melamine and monosaccharides. (A, B) LAESI-MS (ESI solvent was $\mathrm{H}_{2} \mathrm{O}$ ) and HDX-LAESI-MS (ESI solvent was $\mathrm{D}_{2} \mathrm{O}$ ) of an aqueous melamine solution, respectively. (A) Chemical structure of protonated melamine. (C, D) LAESI-MS and HDX-LAESI-MS of hexoses in $20 \times$ diluted (with $\mathrm{H}_{2} \mathrm{O}$ ) orange juice, respectively. (C) Structure of the most abundant (cationized) monosaccharide (D-fructose) in oranges. ${ }^{50}$

Ambient HDX-LAESI-MSI of an Orange Slice and Phalaenopsis Petal. An orange was sliced with a kitchen knife and placed, on a glass slide, onto the temperaturecontrolled sample stage maintained at $4{ }^{\circ} \mathrm{C}$. (HDX-)LAESIMS data were acquired from a $14 \times 17$ pattern $(238$ sample locations) in a sampling area defined from an optical image. The Phalaenopsis petal was mounted with double sided tape on a glass slide and placed onto the sample stage. The Phalaenopsis experiment was imaged in negative ion LAESI mode with $\mathrm{D}_{2} \mathrm{O} / \mathrm{MeOD} 1: 9$ as ESI solvent. Data were acquired from a $14 \times 9$ pattern (126 sample locations) in a sampling area, containing both white and purple patches, defined from an optical image. For both the orange and the petal MSI experiments 20 laser pulses were applied at each location with a frequency of $10 \mathrm{~Hz}$ and $3 \mathrm{~s}$ dwell time per analyses location. The laser spot size was approximately 200 $\mu \mathrm{m}$ in diameter, and the in-between spot interval was $1 \mathrm{~mm}$. These experimental settings resulted in total analysis times of 43 and $20 \mathrm{~min}$, respectively. Proteaplot v2.0.8.5 (Protea Biosciences) was used to create maximum intensity ion maps.

\section{RESULTS AND DISCUSSION}

Ambient HDX-LAESI-MS of Small Molecules in Solution. The feasibility of ambient HDX-LAESI-MS was first investigated in nondeuterated standard solutions with the model compounds melamine, comprising multiple $-\mathrm{NH}$ groups, and monosaccharides, containing multiple $-\mathrm{OH}$ groups in order to observe the effects of HDX on simple molecules containing these widely abundant functional groups that can rapidly exchange protons for deuterons. The selection of these specific two molecules allowed us to study the effect of $\mathrm{HDX}$ on $-\mathrm{OH}$ (shown with monosaccharides) and $-\mathrm{NH}$ (shown with melamine) separately. As $-\mathrm{OH}$ and $-\mathrm{NH}$ moieties are the major source of rapid exchangeable protons in biomolecules, these compounds are considered a good model system for a much wider variety of compounds in life sciences. Melamine has an elemental composition of $\mathrm{C}_{3} \mathrm{~N}_{6} \mathrm{H}_{6}$ and holds six exchangeable $-\mathrm{NH}_{2}$ protons (chemical structure is included in Figure 1A) that should experimentally result in a maximum $m / z$ increase of 7 for the $\left[\mathrm{M}-d_{6}+\mathrm{D}\right]^{+}$ion. Parts A and $\mathrm{B}$ of Figure 1 present LAESI-MS background-subtracted mass spectra of a $100 \mu \mathrm{M}$ aqueous melamine solution with $\mathrm{H}_{2} \mathrm{O}$ (Fig. 1A) and $\mathrm{D}_{2} \mathrm{O}$ (Fig. 1B) as ESI solvents. When $\mathrm{H}_{2} \mathrm{O}$ was used as the ESI solvent an $[\mathrm{M}+\mathrm{H}]^{+}$ion was detected at $m / z$ 127.08. When $\mathrm{D}_{2} \mathrm{O}$ was used as the ESI solvent an $m / z$ distribution from 128.08 to 134.12 was observed. These values correspond to protonated melamine $\left[\mathrm{M}-d_{0}+\mathrm{H}\right]^{+}$and a stepwise increase of the deuterium content up to the completely hydrogen/deuterium (H/D) exchanged value at $m / z 134.12$ for $\left[\mathrm{M}-d_{6}+\mathrm{D}\right]^{+}$. These results correspond nicely with previously reported liquid- and gas-phase melamine HDX studies. ${ }^{28,46}$ Following elemental composition assessment based on accurate mass measurement, a SciFinder elemental composition search was performed to obtain structural isomer options. $\mathrm{C}_{3} \mathrm{~N}_{6} \mathrm{H}_{6}$, initially resulted in 25 different constitutional isomers, however, when only structures comprising six proton exchange sites were included, the number of structure options was substantially reduced to only five, even without any additional MS/MS experiment. The number of proton exchange sites were simply included in the SciFinder search using its "refine by property value" function and selecting " $\mathrm{H}$ Donors".

The most common $\mathrm{C}_{6}$ monosaccharides have an elemental composition of $\mathrm{C}_{6} \mathrm{H}_{12} \mathrm{O}_{6}$ and are detected as sodium adducts in positive mode ESI. ${ }^{47,48}$ Thus, they contain five exchangeable -OH protons (as an example, the structure of a cationized monosaccharide, D-fructose, is included in Figure 1C). As a consequence, $\mathrm{HDX}$ is expected to result in a maximum $\mathrm{m} / \mathrm{z}$ shift of +5 Da. Parts $\mathrm{C}$ and D of Figure 1 depict LAESI-MS (with $\mathrm{H}_{2} \mathrm{O}$ as ESI solvent) and HDX-LAESI-MS (with $\mathrm{D}_{2} \mathrm{O}$ as ESI solvent) background-subtracted mass spectra of $\mathrm{C}_{6}$ monosaccharides in $20 \times$ diluted orange juice. With LAESIMS a single $m / z$ value is detected at 203.05 , corresponding to cationized monosaccharides $[\mathrm{M}+\mathrm{Na}]^{+}$. HDX-LAESI-MS, however, shows a clear increasing isotope pattern ranging from $m / z 203.05$ for $\left[\mathrm{M}-d_{0}+\mathrm{Na}\right]^{+}$up to the fully H/D exchanged at $m / z 208.08$ for $\left[\mathrm{M}-d_{5}+\mathrm{Na}\right]^{+}$. These results are in line with the number of exchanges for the previously observed $\left[\mathrm{M}-d_{5}+\mathrm{D}\right]^{+}$ monosaccharide ion in an atmospheric pressure chemical ionization study when the sample was dissolved and incubated in $\mathrm{D}_{2} \mathrm{O}$ at forehand. ${ }^{49}$ A SciFinder elemental composition search was performed (on $\mathrm{C}_{6} \mathrm{H}_{12} \mathrm{O}_{6}$ ) and 450 results were obtained including all stereoisomers. 95 of the obtained isomers could be eliminated following the selection of structures having five proton exchange sites. Both melamine and monosaccharides demonstrate that ambient HDX-LAESIMS is a simple and expeditious technique for a significant simplification of the structure search for molecules having identical elemental composition and comprising $\mathrm{N}-\mathrm{H}$ or $\mathrm{O}-$ $\mathrm{H}$ bonds. Most importantly, despite the relatively short HDXLAESI process (versus common prolonged sample incubation in deuterated solvents) the exchange still proceeds up to 
completion, yielding great promise for HDX-LAESI-MSI applications in which sample pre-incubation is either impossible or undesired.

Ambient HDX-LAESI-MS of Metabolites in an Orange Slice. An abundant class of biomolecules in fruits are oligosaccharides. Oligosaccharides contain a substantial number of exchangeable protons and are therefore an excellent class of biomolecules to evaluate ambient HDX-LAESI-MS(I) for real biological samples. Oligosaccharides are detected with (HDX-)LAESI-MS in positive ion mode as sodium adducts, so theoretical $\mathrm{m} / z$ values of 365.1054 for $\left[\mathrm{C}_{12} \mathrm{H}_{22} \mathrm{O}_{11}+\mathrm{Na}\right]^{+}$, 527.1583 for $\left[\mathrm{C}_{18} \mathrm{H}_{32} \mathrm{O}_{16}+\mathrm{Na}\right]^{+}, 689.2111$ for $\left[\mathrm{C}_{24} \mathrm{H}_{42} \mathrm{O}_{21}+\right.$ $\mathrm{Na}]^{+}$, etc. are expected to be observed in LAESI-MS experiments of oligosaccharides. With HDX-LAESI-MS these $m / z$ values can be shifted up to a maximum of $5+3 \times(n-1)$, in which $n$ is the number of monomers from $n=1$ onwards, resulting in maximum $m / z$ value shifts upon $\mathrm{HDX}$ of $+8,+11$, +14 , etc. for di-, tri-, and tetrasaccharides, respectively. Figure 2

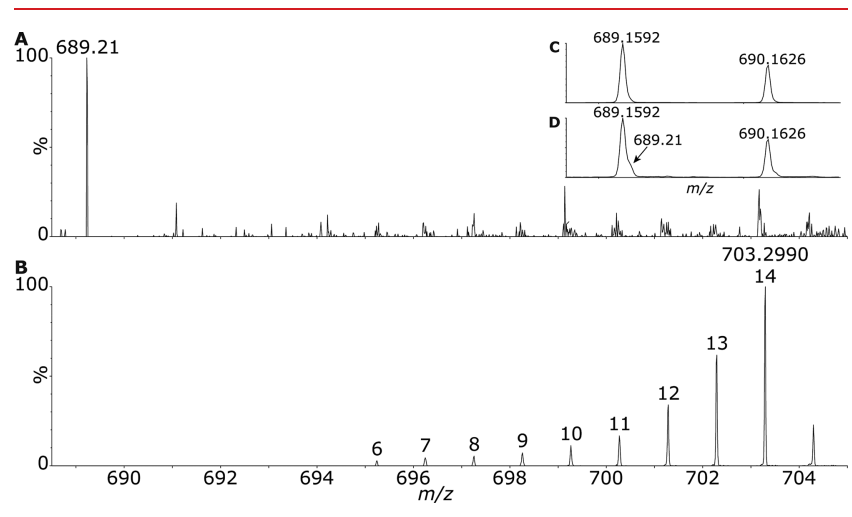

Figure 2. LAESI-TWIM-MS and HDX-LAESI-TWIM-MS of an orange slice. (A, B) IMS drift time 85-92 bins selected and subsequently background-subtracted mass spectra of an orange obtained with (A) LAESI-TWIM-MS and (B) HDX-LAESI-TWIMMS analysis. (A, inset) C shows the ESI solvent background and D shows the orange mass spectrum including background. Obviously, the mass-resolving power is insufficient in $\mathrm{D}$ to baseline separate the cationized oligosaccharide from the background interference.

presents mass spectra of oligosaccharides obtained with LAESI-TWIM-MS and HDX-LAESI-TWIM-MS from the surface of an orange slice. Figure $2 \mathrm{~A}$ shows an $\mathrm{m} / z$ value of 689.21 for $\left[\mathrm{C}_{24} \mathrm{H}_{42} \mathrm{O}_{21}+\mathrm{Na}\right]^{+}$, and Figure $2 \mathrm{~B}$ depicts HDXLAESI-TWIM-MS which clearly shows the expected $m / z$ shift upon HDX for $5+3 \times(4-1)$ of up to +14 , followed by a small ${ }^{13} \mathrm{C}$ isotope peak (Figures S2 and S3 present similar results obtained for other oligosaccharides). The number of observed exchanges in the oligosaccharides is in excellent agreement with previously reported HDX-MALDI and ESI insource HDX-MS studies of oligosaccharides. ${ }^{48,51}$ In addition, note that in Figure $2 \mathrm{~A} \mathrm{~m} / z 689.21$ is located on the tail of an unresolved interfering background ion at $m / z 689.1592$ (mass spectra of ESI solvent background and orange with ESI solvent background are shown in parts $\mathrm{C}$ and $\mathrm{D}$, respectively, of Figure $2)$; therefore, the mass assignment is less accurate and its ${ }^{13} \mathrm{C}$ isotopomer (in Fig 2A) is not observed due to background subtraction. In HDX-LAESI-TWIM-MS, however, the exact $m / z 703.2990$ of $\left[\mathrm{C}_{24} \mathrm{H}_{28} \mathrm{D}_{14} \mathrm{O}_{21}+\mathrm{Na}\right]^{+}$can be obtained without interferences within 5 ppm mass accuracy. This shows that, even when TWIM cannot deal entirely with unresolved background interferences, using HDX can shift the $m / z$ value of a molecule to obtain enhanced mass accuracy.

In principle, the sensitivity of the HDX-LAESI-MS method should be lowered due to additional signals of incomplete $H / D$ exchange (e.g., signals numbered 6-13 in Figure 2B). In this particular example, however, the sensitivity has increased as a result of the $m / z$ shift away from background interferences: the original signal was barely distinguishable from the background signal. On the contrary, it could also happen that due to $\mathrm{H} / \mathrm{D}$ exchange the signal would (partly) overlap with previously notinterfering background or matrix signals. So, any increase or decrease in sensitivity following HDX-LAESI-MS would be strongly analyte, matrix, and background specific.

To demonstrate the added value of HDX for unknown structure identification in biological samples, one out of the many detected ions from the orange slice $(\mathrm{m} / \mathrm{z}$ value 175.1189 , Figure $3 \mathrm{~A}$ ) was used as an example with the current HDX-

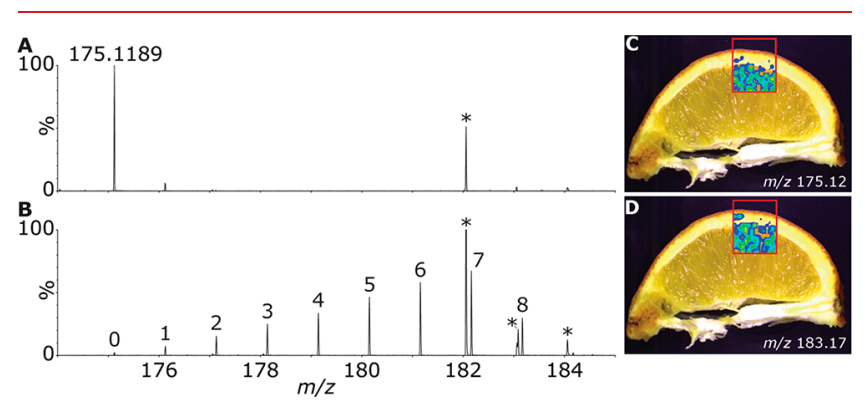

Figure 3. LAESI-MSI and HDX-LAESI-MSI spectra of endogenous arginine in an orange slice. (A, B) Background-subtracted mass spectra of arginine in an orange, obtained with (A) LAESI-MSI and (B) HDX-LAESI-MSI analysis. The ions marked with a * belong to other unknown biomolecules. (C) LAESI-MSI 2D ion map of $\mathrm{m} / \mathrm{z}$ 175.12 for $\left[\mathrm{M}-d_{0}+\mathrm{H}\right]^{+}$. (D) HDX-LAESI-MSI 2D ion map of $\mathrm{m} / z$ 183.17 for $\left[\mathrm{M}-d_{7}+\mathrm{D}\right]^{+}$. The red box on the superimposed pictures marks the imaged area.

LAESI approach. This ion at $m / z 175.1189$ relates to an endogenous compound and provides a nice example that HDX can strongly facilitates its structure elucidation. Based on the exact mass only one elemental composition (comprising: $\left.\mathrm{C}_{0-20}, \mathrm{H}_{0-50}, \mathrm{O}_{0-10}, \mathrm{~N}_{0-10}, \mathrm{~S}_{0-2}, \mathrm{P}_{0-2}, \mathrm{Na}_{0-1}, \mathrm{~K}_{0-1}\right)$ was obtained within $10 \mathrm{ppm}$ (3.4 ppm) mass accuracy: $\mathrm{C}_{6} \mathrm{H}_{15} \mathrm{~N}_{4} \mathrm{O}_{2}$, so obviously a protonated substance rather than a cationized species. A SciFinder elemental composition search, excluding substances comprising isotopes and substances without any references, resulted in 103 chemical structures. HDX-LAESI-MS showed up to eight exchanges to occur (Figure 3B), including the deuteration. Due to the seven detected exchangeable protons for $\left[\mathrm{M}-d_{7}+\mathrm{D}\right]^{+}$the search was refined to only include compounds comprising seven proton donors. As a result, from the initial 103 options only 13 structures fulfill this requirement (Table S1), including several stereoisomers. Upon excluding individual stereoisomers, which cannot be resolved by MS alone, 10 out of the remaining 11 substances have only $1-5$ references according to Scifinder with various origins such as, e.g., synthesis, patents, and review articles. One candidate substance, however, is arginine, which is obviously well documented with approximately 100000 references. This thus induces the hypothesis that the ion in the orange slice belongs to arginine. Instead of verification (MS/ MS) experiments on all the 103 options prior to HDX, the experimental verification could-due to the large reduction of 

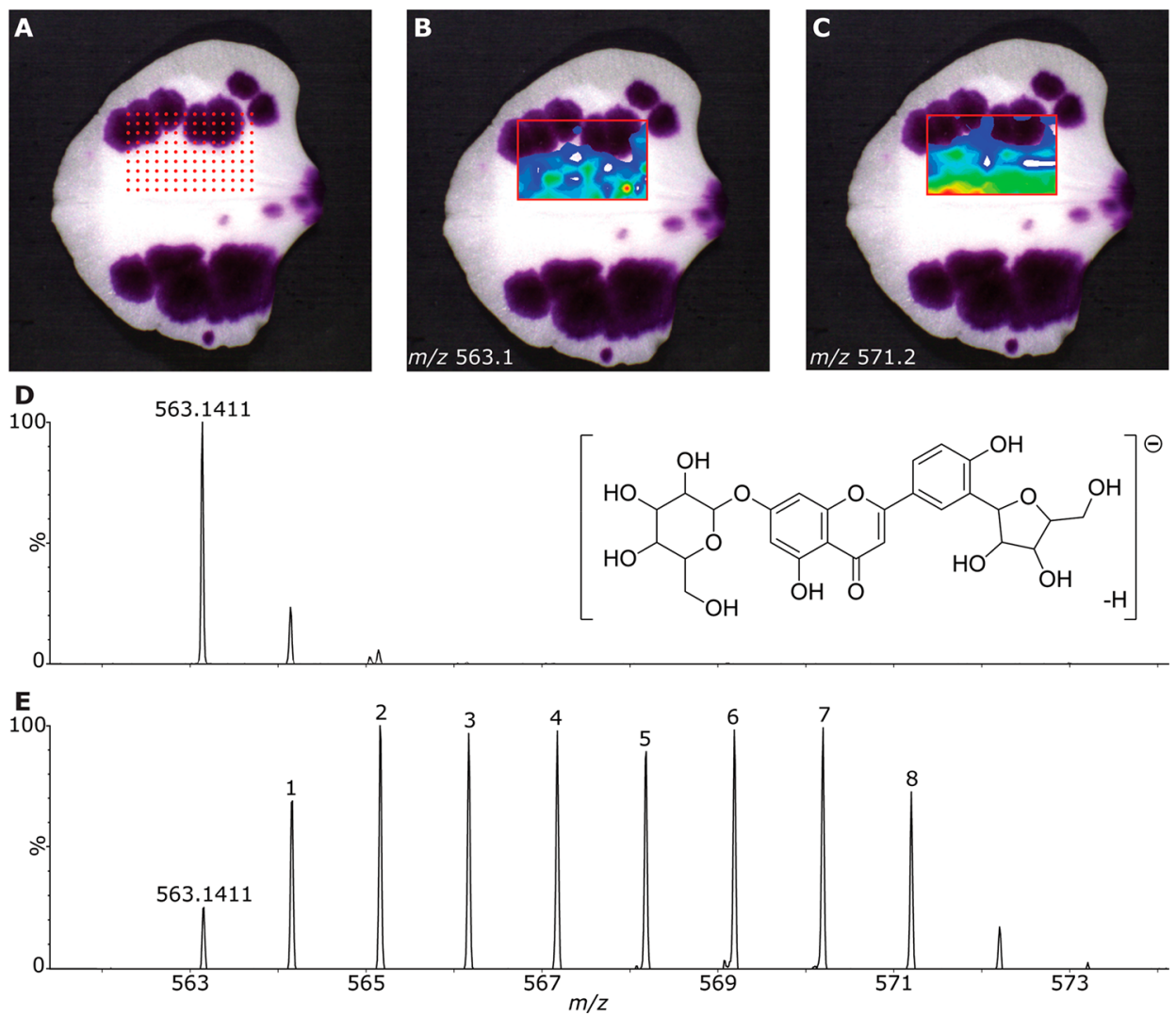

Figure 4. (HDX-)LAESI-MSI of a spatially resolved apigenin-derived flavonoid outside the purple patches of a Phalaenopsis petal. ${ }^{53}$ (A) Laser $x-y$ locations (red dots) on the optical image. (B) 2D ion map of $m / z 563.14 \pm 0.01$ for $\left[\mathrm{M}-d_{0}-\mathrm{H}\right]^{-}$. (C) $2 \mathrm{D}$ ion map of $\mathrm{m} / z 571.19 \pm 0.01 \mathrm{for}$ the fully exchanged $\left[\mathrm{M}-d_{8}-\mathrm{H}\right]^{-}$. The red box on the superimposed pictures marks the imaged area. (D) LAESI-MS background-subtracted mass spectrum and the molecular structure of the apigenin derived flavonoid comprising eight exchangeable protons for $[\mathrm{M}-\mathrm{H}]^{-}$. (E) HDX-LAESI-MS background-subtracted mass spectrum. A larger $m / z$ range and MS/MS mass spectra for D and E are presented in Figures S5 and S6, respectively.

options following HDX-be achieved by comparison of the orange sample with only an arginine standard solution. The obtained (HDX-)LAESI-MS/MS fragmentation spectra of $\mathrm{m} / z$ $175.1\left(\mathrm{H}_{2} \mathrm{O}\right)$ and $m / z 183.2\left(\mathrm{D}_{2} \mathrm{O}\right)$ are presented in Figure $\mathrm{S} 4$. The arginine reference solution and the orange sample show similar fragmentation spectra, and both nicely correspond with literature. $^{52}$ Furthermore, the proposed elemental compositions for the observed neutral losses from $\mathrm{m} / z 175.1$ and 183.2 are confirmed by the corresponding number of $\mathrm{H}$ and $\mathrm{D}$ atoms, thus providing additional evidence that the detected $m$ / $z$ value 175.1198 is indeed the amino acid arginine (Figure S4). These results show that the developed HDX approach is also applicable to endogenous amino acids present in a real sample matrix.

As an initial imaging feasibility experiment, (HDX-)LAESIMSI was performed on the same orange slice. The laser ablation $x-y$ coordinates were used to create $2 \mathrm{D}$ ion maps of arginine and the fully $\mathrm{H} / \mathrm{D}$ exchanged arginine LAESI-MSI signals, and both were superimposed on an optical image of the orange (Figure $3 \mathrm{C}, \mathrm{D})$. The area inside the red box was imaged and for both ions, $m / z 175.12$ for $\left[\mathrm{M}-d_{0}+\mathrm{H}\right]^{+}$and $m / z 183.17$ for $\left[\mathrm{M}-d_{7}+\mathrm{D}\right]^{+}$, images were only obtained in the pulp area of the orange slice and not on the peel, in accordance with expectations (apart from a few minor slicing artifacts due to orange juice wetting the knife's edge).

Ambient HDX-LAESI-MSI of (Secondary) Metabolites in a Phalaenopsis Petal. In order to show the potential of ambient untargeted HDX-LAESI-MSI in plant metabolomics, a
Phalaenopsis petal sample was imaged for secondary plant metabolites. Luteolin-type flavonoids are expected to show colocalization with anthocyanins (purple pigments), whereas apigenin-based flavonoids are reported be absent in these purple regions. ${ }^{53}$ Figure 4 presents the $2 \mathrm{D}$ ion maps and (HDX-)LAESI-MSI spectra of an apigenin-based flavonoid. Figure $4 \mathrm{~A}$ presents an optical image of the petal including the laser $x-y$ locations marked as red dots, whereas Figure 4B,C shows the apigenin-derived flavonoid LAESI-MSI, $m / z 563.1$ for $[\mathrm{M}-\mathrm{H}]^{-}$, and HDX-LAESI-MSI, $m / z 571.2$ for $\left[\mathrm{M}-d_{8}-\mathrm{H}\right]^{-}$, $2 \mathrm{D}$ ion maps superimposed on this optical image, respectively. Indeed, the apigenin-type flavonoid shows an inversedistribution with the purple pigments in the petal, demonstrating that similar ion distributions are obtained with both LAESI-MSI and HDX-LAESI-MSI experiments. The obtained (HDX-)LAESI-MSI mass spectra are presented in Figure 4D,E. The number of $\mathrm{H} / \mathrm{D}$ exchanges (Figure $4 \mathrm{E}$ ) are clearly observed to be 8 , with the small signal at $m / z 572.2$ to be a ${ }^{13} \mathrm{C}$ isotope in a similar ratio as the (LAESI-MSI) isotope signal observed in Figure 4D. Figure 4D also shows the proposed molecular structure, comprising eight exchangeable protons for $[\mathrm{M}-\mathrm{H}]^{-}$(please note that the positions of the glycoside bonds are putative), and nicely corresponds with literature. ${ }^{53}$ As the data was recorded in an untargeted approach, many signals were obtained and a larger $m / z$ range of the analysis is provided in Figure S5. One of the observed $\mathrm{m} / z$ values, 609.1480 , relates to a luteolin-type flavonoid and was processed similarly as the apigenin-type flavonoid (Figure 5). 


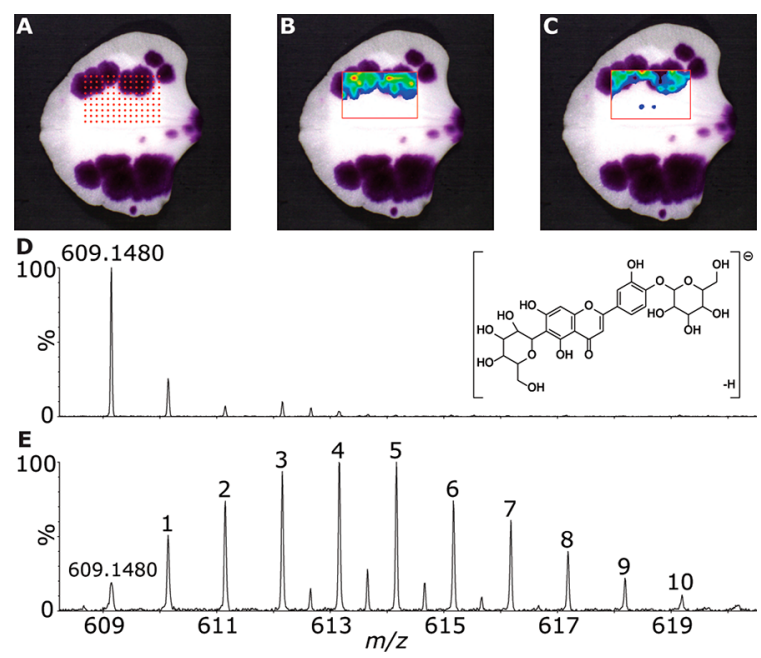

Figure 5. (HDX-)LAESI-MSI of a spatially resolved luteolin diglycoside present in the purple patches of a Phalaenopsis petal. (A) Laser $x-y$ locations (red dots) on the optical image. (B) 2D ion map of $m / z 609.15 \pm 0.01$ for $\left[\mathrm{M}-d_{0}-\mathrm{H}\right]^{-}$. (C) $2 \mathrm{D}$ ion map of $m / z$ $619.19 \pm 0.01$ for the fully exchanged $\left[\mathrm{M}-d_{10}-\mathrm{H}\right]^{-}$. The red box on the superimposed pictures marks the imaged area. (D) LAESI-MS background-subtracted mass spectrum and the molecular structure of the luteolin diglycoside comprising 10 exchangeable protons for $[\mathrm{M}-$ $\mathrm{H}]^{-}$. (E) HDX-LAESI-MS background-subtracted mass spectrum. A larger $m / z$ range for $\mathrm{D}$ and $\mathrm{E}$ is presented in Figure S5.

As expected, instead of an inverse-localization the resulting 2D ion maps (Figures 5A-C) now showed co-localization with the purple patches of the petal. Additionally, also these (HDX)LAESI-MSI mass spectra (Figure 5D,E) show the proposed molecular structure (Fig 5D) and an up to fully $\mathrm{H} / \mathrm{D}$ exchanged isotope distribution (Figure 5E). Both Figures 4 and 5 thus present $2 \mathrm{D}$ ion maps corresponding with the colocalization and distribution of the compounds within (Figure 5 ) or outside (Figure 4) the purple patches for both LAESIMSI as well as HDX-LAESI-MSI, demonstrating the feasibility of the HDX-MSI approach.

The larger $m / z$ range presented in Figure S5 shows that untargeted HDX-LAESI-MS(I) is suitable especially for the more abundant analytes in a sample. Analytes with a low signal-to-noise ratio could, however, be concealed by matrix or background signals. It is therefore recommended to compare both LAESI-MS(I) with HDX-LAESI-MS(I) for each sample to extract the highest amount of information. Additionally, in case the number of $\mathrm{H} / \mathrm{D}$ exchanges would be unclear, either due to interfering signals, incomplete exchange or limited ion abundance; as might be observed in Figure 5E, a minimum number of exchanges (e.g., 10 in Fig $5 \mathrm{E}$ ) could still be used to reduce the number of structural options in a database search.

Finally, targeted (HDX-)LAESI-MS/MS experiments for the identification of the apigenin-type flavonoid were performed on the white areas of the petal, and the obtained spectra are shown in Figure S6. The proposed molecular structure (Fig. 4D) showed apigenin with an O-glycoside as well as a Cglycoside modification. The MS/MS spectra indeed show the $\mathrm{m} / z$ values of apigenin itself (the aglycon) at 269.0454 for [M$\left.d_{0}-\mathrm{H}\right]^{-}$(Figure S6A) and the expected $2 \mathrm{H} / \mathrm{D}$ exchanges to obtain $m / z 271.0581$ for $\left[\mathrm{M}-d_{2}-\mathrm{H}\right]^{-}$(Figure S6B). Also, the presence of an $\mathrm{O}$-glycoside was confirmed by the neutral loss of $162.0531\left(\mathrm{C}_{6} \mathrm{H}_{10} \mathrm{O}_{5}\right)$ showing $m / z 401.0877$ (Figure $\mathrm{S} 6 \mathrm{~A}$ ) and, upon HDX-MSI, $165.0715\left(\mathrm{C}_{6} \mathrm{H}_{7} \mathrm{D}_{3} \mathrm{O}_{5}\right)$ showing $\mathrm{m} / z$
406.1202 (Figure S6B). Proposed (fragment) structures for $\mathrm{m} /$ $z$ 563.1408, 401.0877, and 269.0454 as well as 571.1917, 406.1202, and 271.0581 are provided in Figure S7. CGlycosides (in contrast to O-glycosides) are known to show several glycoside ring cleavages in MS/MS experiments. ${ }^{54-56}$ These cleavages were also observed by both the deuterated as well as the nondeuterated precursors and are presented (along with the O-glycoside and the apigenin fragments) in Table S2. The apigenin-type flavonoid, comprising apigenin with $\mathrm{O}$ glycoside and C-glycoside modifications, analyzed direct from plant tissue, could be observed and additionally confirmed by the mass difference between neutral losses and H/D exchanged neutral losses. These experiments clearly show the feasibility and benefit of HDX-LAESI-MSI for structure elucidation in spatially resolved plant metabolomics.

\section{CONCLUSIONS}

Here, we developed a novel ambient HDX-LAESI-MSI approach to acquire additional molecular structural information for the identification and confirmation of (bio)molecules. Like all ambient MS approaches, this method provides a simple and rapid analysis of samples under ambient conditions but now with the benefit of trivially obtainable additional chemical structural information. Ambient HDX-LAESI-MS in nondeuterated solutions of the model compounds melamine and monosaccharides revealed that information on the maximum number of $\mathrm{H} / \mathrm{D}$ exchanges significantly reduced the number of structures in elemental composition database searches. Mass spectrometric investigation of an orange slice revealed the presence of, among others, oligosaccharides, and the amino acid arginine. The latter was investigated in detail, and it was proven that combining accurate mass, MS/MS, and literature data with HDX led to the unambiguous structural elucidation of that compound. Additionally, a significant increase in mass accuracy was observed for an oligosaccharide as a result of the HDX reaction $m / z$ shift away from an unresolved background interference. The feasibility of the method in support of structure elucidation in ambient MSI of (secondary) plant metabolites was clearly demonstrated in an orange slice and a Phalaenopsis petal. As a future hardware simplification, the use of a dual or $\theta$ ESI sprayer-one with $\mathrm{H}_{2} \mathrm{O}$ and the other with $\mathrm{D}_{2} \mathrm{O}-$ can be considered in the LAESI set-up.

\section{ASSOCIATED CONTENT}

\section{Supporting Information}

The Supporting Information is available free of charge at https://pubs.acs.org/doi/10.1021/jasms.9b00082.

Figures and tables showing additional MS data (PDF)

\section{AUTHOR INFORMATION}

\section{Corresponding Author}

*E-mail: michel.nielen@wur.nl.

ORCID $\odot$

Maurice C. R. Franssen: 0000-0002-3615-9115

Han Zuilhof: 0000-0001-5773-8506

Michel W. F. Nielen: 0000-0003-4634-0249

Notes

The authors declare no competing financial interest. 


\section{ACKNOWLEDGMENTS}

The authors are grateful to receive funding for this research from The Netherlands Organization for Scientific Research (NWO) in the framework of the Technology Area TACOAST2 of the Fund New Chemical Innovations (Project No. 053.21.111). Pepijn Beekman and Sevil Sahin are acknowledged for inspiring conversations.

\section{REFERENCES}

(1) Bowman, A. P.; Heeren, R. M. A.; Ellis, S. R. Advances in mass spectrometry imaging enabling observation of localised lipid biochemistry within tissues. TrAC, Trends Anal. Chem. 2019, 120, 115197.

(2) Lamont, L.; Eijkel, G. B.; Jones, E. A.; Flinders, B.; Ellis, S. R.; Porta Siegel, T.; Heeren, R. M. A.; Vreeken, R. J. Targeted drug and metabolite imaging: desorption electrospray ionization combined with triple quadrupole mass spectrometry. Anal. Chem. 2018, 90 (22), 13229-13235.

(3) McDonnell, L. A.; Heeren, R. M. A. Imaging mass spectrometry. Mass Spectrom. Rev. 2007, 26 (4), 606-643.

(4) Caprioli, R. M. Imaging mass spectrometry: molecular microscopy for enabling a new age of discovery. Proteomics 2014, 14 (7-8), 807-809.

(5) Dong, Y.; Li, B.; Malitsky, S.; Rogachev, I.; Aharoni, A.; Kaftan, F.; Svatoš, A.; Franceschi, P. Sample preparation for mass spectrometry imaging of plant tissues: a review. Front. Plant Sci. 2016, 7, 60 .

(6) Dettmer, K.; Aronov, P. A.; Hammock, B. D. Mass spectrometrybased metabolomics. Mass Spectrom. Rev. 2007, 26 (1), 51-78.

(7) Wu, C.; Dill, A. L.; Eberlin, L. S.; Cooks, R. G.; Ifa, D. R. Mass spectrometry imaging under ambient conditions. Mass Spectrom. Rev. 2013, 32 (3), 218-243.

(8) Cha, S.; Zhang, H.; Ilarslan, H. I.; Wurtele, E. S.; Brachova, L.; Nikolau, B. J.; Yeung, E. S. Direct profiling and imaging of plant metabolites in intact tissues by using colloidal graphite-assisted laser desorption ionization mass spectrometry. Plant J. 2008, 55 (2), 348360.

(9) Thomas, A.; Charbonneau, J. L.; Fournaise, E.; Chaurand, P. Sublimation of new matrix candidates for high spatial resolution imaging mass spectrometry of lipids: enhanced information in both positive and negative polarities after 1,5-diaminonapthalene deposition. Anal. Chem. 2012, 84 (4), 2048-2054.

(10) Cooks, R. G.; Ouyang, Z.; Takats, Z.; Wiseman, J. M. Ambient mass spectrometry. Science 2006, 311 (5767), 1566-1570.

(11) Cody, R. B.; Laramée, J. A.; Durst, H. D. Versatile new ion source for the analysis of materials in open air under ambient conditions. Anal. Chem. 2005, 77 (8), 2297-2302.

(12) Venter, A.; Nefliu, M.; Cooks, R. G. Ambient desorption ionization mass spectrometry. TrAC, Trends Anal. Chem. 2008, 27 (4), 284-290.

(13) Chen, H.; Gamez, G.; Zenobi, R. What can we learn from ambient ionization techniques? J. Am. Soc. Mass Spectrom. 2009, 20 (11), 1947-1963.

(14) Perez, C. J.; Bagga, A. K.; Prova, S. S.; Yousefi Taemeh, M.; Ifa, D. R. Review and perspectives on the applications of mass spectrometry imaging under ambient conditions. Rapid Commun. Mass Spectrom. 2019, 33, 27-53.

(15) Manicke, N. E.; Dill, A. L.; Ifa, D. R.; Cooks, R. G. Highresolution tissue imaging on an orbitrap mass spectrometer by desorption electrospray ionization mass spectrometry. J. Mass Spectrom. 2010, 45 (2), 223-226.

(16) Little, J. L.; Cleven, C. D.; Brown, S. D. Identification of "known unknowns" utilizing accurate mass data and chemical abstracts service databases. J. Am. Soc. Mass Spectrom. 2011, 22 (2), 348-359.

(17) Cajka, T.; Fiehn, O. LC-MS-based lipidomics and automated identification of lipids using the lipidblast in-silico MS/MS Library. Methods Mol. Biol. 2017, 1609 (1609), 149-170.
(18) Li, H.; Smith, B. K.; Márk, L.; Nemes, P.; Nazarian, J.; Vertes, A. Ambient molecular imaging by laser ablation electrospray ionization mass spectrometry with ion mobility separation. Int. J. Mass Spectrom. 2015, 377, 681-689.

(19) Bennett, R. V.; Gamage, C. M.; Galhena, A. S.; Fernández, F. M. Contrast-enhanced differential mobility-desorption electrospray ionization-mass spectrometry imaging of biological tissues. Anal. Chem. 2014, 86 (8), 3756-3763.

(20) van Geenen, F. A. M. G.; Franssen, M. C. R.; Zuilhof, H.; Nielen, M. W. F. Reactive laser ablation electrospray ionization timeresolved mass spectrometry of click reactions. Anal. Chem. 2018, 90, 10409-10416.

(21) Chen, H.; Cotte-Rodriguez, I.; Cooks, R. G. cis-Diol functional group recognition by reactive desorption electrospray ionization (DESI). Chem. Commun. 2006, 6, 597-599.

(22) Kostyukevich, Y.; Acter, T.; Zherebker, A.; Ahmed, A.; Kim, S.; Nikolaev, E. Hydrogen/deuterium exchange in mass spectrometry. Mass Spectrom. Rev. 2018, 37 (6), 811-853.

(23) Masson, G. R.; Jenkins, M. L.; Burke, J. E. An overview of hydrogen deuterium exchange mass spectrometry (HDX-MS) in drug discovery. Expert Opin. Drug Discovery 2017, 12 (10), 981-994.

(24) Adejare, A.; Brown, P. W. Hydrogen/deuterium exchange to differentiate fragment ions from pseudomolecular ions by electrospray tandem mass spectrometry. Anal. Chem. 1997, 69 (8), 1525-1529.

(25) Cheng, X.; Fenselau, C. Hydrogen/deuterium exchange of mass-selected peptide ions with $\mathrm{ND}_{3}$ in a tandem sector mass spectrometer. Int. J. Mass Spectrom. Ion Processes 1992, 122, 109-119.

(26) Hunt, D. F.; McEwen, C. N.; Upham, R. A. Determination of active hydrogen in organic compounds by chemical ionization mass spectrometry. Anal. Chem. 1972, 44 (7), 1292-1294.

(27) Chen, H.; Zheng, J.; Zhang, X.; Luo, M.; Wang, Z.; Qiao, X. Surface desorption atmospheric pressure chemical ionization mass spectrometry for direct ambient sample analysis without toxic chemical contamination. J. Mass Spectrom. 2007, 42 (8), 1045-1056.

(28) Vail, T. M.; Jones, P. R.; Sparkman, O. D. Rapid and unambiguous identification of melamine in contaminated pet food based on mass spectrometry with four degrees of confirmation. $J$. Anal. Toxicol. 2007, 31 (6), 304-312.

(29) Nemes, P.; Vertes, A. Laser ablation electrospray ionization for atmospheric pressure, in vivo, and imaging mass spectrometry. Anal. Chem. 2007, 79 (21), 8098-8106.

(30) Nemes, P.; Barton, A. A.; Li, Y.; Vertes, A. Ambient molecular imaging and depth profiling of live tissue by infrared laser ablation electrospray ionization mass spectrometry. Anal. Chem. 2008, 80 (12), $4575-4582$.

(31) Vertes, A.; Nemes, P.; Shrestha, B.; Barton, A. A.; Chen, Z.; Li, Y. Molecular imaging by Mid-IR laser ablation mass spectrometry. Appl. Phys. A: Mater. Sci. Process. 2008, 93 (4), 885-891.

(32) Sripadi, P.; Nazarian, J.; Hathout, Y.; Hoffman, E. P.; Vertes, A. In vitro analysis of metabolites from the untreated tissue of Torpedo californica electric organ by mid-infrared laser ablation electrospray ionization mass spectrometry. Metabolomics 2009, 5 (2), 263-276.

(33) Nemes, P.; Barton, A. A.; Vertes, A. Three-dimensional imaging of metabolites in tissues under ambient conditions by laser ablation electrospray ionization mass spectrometry. Anal. Chem. 2009, 81 (16), $6668-6675$.

(34) Nemes, P.; Vertes, A. Laser ablation electrospray ionization for atmospheric pressure molecular imaging mass spectrometry. Methods Mol. Biol. 2010, 656 (656), 159-171.

(35) Nielen, M. W. F.; van Beek, T. A. Macroscopic and microscopic spatially-resolved analysis of food contaminants and constituents using laser-ablation electrospray ionization mass spectrometry imaging. Anal. Bioanal. Chem. 2014, 406 (27), 6805-6815.

(36) van Geenen, F. A. M. G.; Franssen, M. C. R.; Schotman, A. H. M.; Zuilhof, H.; Nielen, M. W. F. Ambient characterization of synthetic fibers by laser ablation electrospray ionization mass spectrometry. Anal. Chem. 2017, 89 (7), 4031-4037. 
(37) Shrestha, B.; Vertes, A. In situ metabolic profiling of single cells by laser ablation electrospray ionization mass spectrometry. Anal. Chem. 2009, 81 (20), 8265-8271.

(38) Shrestha, B.; Vertes, A. Direct analysis of single cells by mass spectrometry at atmospheric pressure. J. Visualized Exp. 2010, No. 43, e2144.

(39) Shrestha, B.; Patt, J. M.; Vertes, A. In situ cell-by-cell imaging and analysis of small cell populations by mass spectrometry. Anal. Chem. 2011, 83 (8), 2947-2955.

(40) Nazari, M.; Ekelöf, M.; Khodjaniyazova, S.; Elsen, N. L.; Williams, J. D.; Muddiman, D. C. Direct screening of enzyme activity using infrared matrix-assisted laser desorption electrospray ionization. Rapid Commun. Mass Spectrom. 2017, 31 (22), 1868-1874.

(41) van Geenen, F. A. M. G.; Franssen, M. C. R.; Miikkulainen, V.; Ritala, M.; Zuilhof, H.; Kostiainen, R.; Nielen, M. W. F. $\mathrm{TiO}_{2}$ photocatalyzed oxidation of drugs studied by laser ablation electrospray ionization mass spectrometry. J. Am. Soc. Mass Spectrom. 2019, 30, 639-646.

(42) Kostyukevich, Y.; Kononikhin, A.; Popov, I.; Nikolaev, E. Simple atmospheric hydrogen/deuterium exchange method for enumeration of labile hydrogens by electrospray ionization mass spectrometry. Anal. Chem. 2013, 85 (11), 5330-5334.

(43) Katta, V.; Chait, B. T. Hydrogen/deuterium exchange electrospray ionization mass spectrometry: a method for probing protein conformational changes in solution. J. Am. Chem. Soc. 1993, 115 (14), 6317-6321.

(44) Kim, H. J.; Liyanage, O. T.; Mulenos, M. R.; Gallagher, E. S. Mass spectral detection of forward- and reverse-hydrogen/deuterium exchange resulting from residual solvent vapors in electrospray sources. J. Am. Soc. Mass Spectrom. 2018, 29 (10), 2030-2040.

(45) Jansson, E. T.; Lai, Y.-H.; Santiago, J. G.; Zare, R. N. Rapid hydrogen-deuterium exchange in liquid droplets. J. Am. Chem. Soc. 2017, 139 (20), 6851-6854.

(46) Nielen, M. W. F.; van de Ven, H. J. F. M. Characterization of (methoxymethyl)melamine resin by combined chromatographic/ mass spectrometric techniques. Rapid Commun. Mass Spectrom. 1996, 10 (1), 74-81.

(47) Kostyukevich, Y.; Kononikhin, A.; Popov, I.; Nikolaev, E. InESI source hydrogen/deuterium exchange of carbohydrate ions. Anal. Chem. 2014, 86 (5), 2595-2600.

(48) Price, N. P. J. Oligosaccharide structures studied by hydrogendeuterium exchange and MALDI-TOF mass spectrometry. Anal. Chem. 2006, 78 (15), 5302-5308.

(49) Choi, S. S.; Kim, J. C. Deuterium effect on ionization and fragmentation patterns of monosaccharides ionized by atmospheric pressure chemical ionization. Carbohydr. Res. 2010, 345 (3), 408413.

(50) Carballo, S.; Zingarello, F. A.; Maestre, S. E.; Todolí, J. L.; Prats, M. S. Optimisation of analytical methods for the characterisation of oranges, clementines and citrus hybrids cultivated in Spain on the basis of their composition in ascorbic acid, citric acid and major sugars. Int. J. Food Sci. Technol. 2014, 49 (1), 146-152.

(51) Kostyukevich, Y.; Kononikhin, A.; Popov, I.; Nikolaev, E. Conformations of cationized linear oligosaccharides revealed by FTMS combined with in-ESI H/D exchange. J. Mass Spectrom. 2015, 50 (10), 1150-1156.

(52) Shek, P. Y. I.; Zhao, J.; Ke, Y.; Siu, K. W. M.; Hopkinson, A. C. Fragmentations of protonated arginine, lysine and their methylated derivatives: concomitant losses of carbon monoxide or carbon dioxide and an amine. J. Phys. Chem. A 2006, 110 (27), 8282-8296.

(53) Etalo, D. W.; De Vos, R. C. H.; Joosten, M. H. A. J.; Hall, R. D. Spatially resolved plant metabolomics: some potentials and limitations of laser-ablation electrospray ionization mass spectrometry metabolite imaging. Plant Physiol. 2015, 169 (3), 1424-1435.

(54) Figueirinha, A.; Paranhos, A.; Pérez-Alonso, J. J.; Santos-Buelga, C.; Batista, M. T. Cymbopogon citratus leaves: characterization of flavonoids by HPLC-PDA-ESI/MS/MS and an approach to their potential as a source of bioactive polyphenols. Food Chem. 2008, 110 (3), $718-728$.
(55) Zhang, M.; Duan, C.; Zang, Y.; Huang, Z.; Liu, G. The flavonoid composition of flavedo and juice from the pummelo cultivar (Citrus grandis (L.) Osbeck) and the grapefruit cultivar (Citrus paradisi) from China. Food Chem. 2011, 129 (4), 1530-1536.

(56) Ibrahim, R. M.; El-Halawany, A. M.; Saleh, D. O.; Naggar, E. M. B. E.; El-Shabrawy, A. E.-R. O.; El-Hawary, S. S. HPLC-DAD-MS/ MS profiling of phenolics from Securigera securidaca flowers and its anti-hyperglycemic and anti-hyperlipidemic activities. Rev. Bras. Farmacogn. 2015, 25 (2), 134-141. 